\title{
6. Implikationen unsicherer Iterationsauszahlungen auf die verhaltensorientierte Realoptionsbewertung
}

In der Modellierung wurde davon ausgegangen, dass die Iterationsauszahlungen sicher und konstant sind. Allerdings dürfte diese Annahme in den meisten Projekten nicht erfüllt sein. ${ }^{183} \mathrm{Um}$ das Modell realistischer zu gestalten, wäre es also sinnvoll, das Modell um die Annahme stochastischer Iterationsauszahlungen zu erweitern. Die bisherige Vorgehensweise lässt sich prinzipiell auch auf stochastische Iterationsauszahlungen übertragen. Dabei ist zunächst zu fragen, welcher stochastische Prozess geeignet ist, um die Entwicklung der Iterationsauszahlungen adäquat beschreiben zu können. ${ }^{184}$ Im Zuge dessen sind auch Korrelationsannahmen zwischen den Projekteinzahlungen und den Projektauszahlungen zu treffen. Die Abbildung der Korrelationen kann dabei analog zur vorgestellten Berücksichtigung der kontemporären Unsicherheit erfolgen. Darüber hinaus wären die kritischen Werte anzupassen, wobei die bisher vorgestellten Modelle dann nicht mehr geeignet sind. In diesem Fall wäre das Modell von Carr bzw. das Modell von Margrabe heranzuziehen, da diese Modelle von stochastischen Auszahlungen ausgehen und auch Korrelationen erfassen können. ${ }^{185}$ Die stochastischen Auszahlungen wirken sich auch auf den Optimierungsansatz bezüglich des Glättungsparameters aus. Dabei wäre allerdings zu prüfen, ob effizientere Lösungsmöglichkeiten des Optimierungsproblems bestehen, da ansonsten keine flexiblen Analysen möglich sind. Davon abgesehen könnten die hier vorgestellten kognitiven Verzerrungen dann unter Einbezug stochastischer Iterationsauszahlungen untersucht werden. Allerdings könnten sich aus der Existenz stochastischer Auszahlungen Ansatzpunkte für weitere kognitive Verzerrungen ergeben. Stochastische Auszahlungen dürften jedoch anders zu behandeln sein als stochastische Einzahlungen. So dürfte sich bei stochastischen Auszahlungen keine Notwendigkeit zur Modellierung einer kontemporären Unsicherheit ergeben, da wohl davon ausgegangen werden kann, dass den Entscheidungsträgern nach jeder Iteration die angefallenen Auszahlungen der letzten Iteration bekannt sind. Kognitive Verzerrungen sind daher eher bei der Prognose der noch ausstehenden Iterationsauszahlungen zu erwarten, wobei diese Prognosen beispielsweise optimistisch oder pessimistisch verzerrt sein könnten. ${ }^{186}$ Insofern könnte es sich anbieten, die Prognosen der Entscheidungsträger zu modellieren, um anschließend die Auswirkungen auf die Realoptionswerte analysieren zu können. Allerdings wäre dann wohl die Entscheidungslogik dahingehend anzupassen, dass die Entscheidungsträger ihre Abbruch- bzw. Fortführungsentscheidungen nicht mehr auf der Basis optionspreistheoretischer Modelle, sondern auf der Basis ihrer Prognosen treffen.

\footnotetext{
183 vgl. auch folgend Baumeister/llg [Agile Software Development] 8

184 vgl. Müller et al. [Investment Projects] 385

185 vgl. Taudes [Growth Options] $174 \mathrm{f}$.

186 vgl. Helpenstein [Managementprognosen] $89 \mathrm{f}$.
}

(C) Springer Fachmedien Wiesbaden GmbH, ein Teil von Springer Nature 2019

M. Wittke, Verhaltensorientierte Realoptionsbewertung von Investitionsprojekten, BestMasters, https://doi.org/10.1007/978-3-658-24750-8_6 